\title{
Technology Architecture of Intelligent Remanufacturing
}

\author{
Ziqiang Zhou, Guohong Dai*, Chaobin Hu, Xiangyan Zhang \\ Jiangsu Key Laboratory of Recycling and Reuse Technology for Mechanical and Electronic Products \\ Changshu Institute of Technology \\ Changshu, China \\ *dgh@cslg.edu.cn
}

\begin{abstract}
With the attention of world manufacturing paid to sustainable development, the social value of recycling and reusing waste products has been generally recognized. The disassembly and remanufacturing of waste products or parts are very important aspects. Only by improving the productivity of remanufacturing waste products can the effective recycling and reuse of waste products be guaranteed. In this study, the intelligent remanufacturing technical system is proposed by analyzing the remanufacturing process system, combined with the ideas and knowledge of intelligent manufacturing. Three aspects are demonstrated, including reverse supply chain, remanufacturing enterprise management, and the application method of artificial intelligence technology in remanufacturing equipment. The architecture and development of intelligent remanufacturing are generally demonstrated, and the issues related to intelligent remanufacturing are finally discussed.
\end{abstract}

Keywords-remanufacturing; intelligent manufacturing; knowledge system; recycling; end of life product

\section{INTRODUCTION}

With the proposal of the concepts of "Made in China 2025" and "Industry 4.0", intelligent manufacturing (IM), as a new issue, becomes a novelty in the whole manufacturing industry. Actually, IM is not a new thing. The concept of IM has been proposed in the 1990s. The basic idea is to use relevant technologies of artificial intelligence to assist the reasoning, judgment, and decision making in manufacturing process, improving the technical level and efficiency of manufacturing industry. The key technologies include machine learning, intelligent decision, and intelligent optimization algorithms. Especially for the recent 10 years, intelligent algorithms including ant colony algorithm, particle swarm optimization, support vector machine, as well as self-learning algorithms have been significantly developed and feasibly applied in every field of social development, thus providing the theoretical and technical support for the further development of IM.

Remanufacturing, as an important branch of the whole manufacturing industry, is the symbolization of sustainable development idea in manufacturing industry. It refers to the technical process using waste parts as objects and restoring their original sizes, or even their service performance [1]. Remanufacturing is of great significance for easing the shortage of resources, and protecting the environment. However, compared with general product manufacturing, there are many special features in remanufacturing process, such as the dispersion of quality and sources of objects to be manufactured and the uncertainty of the process parameters. Due to these features, the study and application of intelligent remanufacturing (IR) has far-reaching significance.

\section{TECHNOLOGY PROCESS ANALYSIS OF REMANUFACTURING}

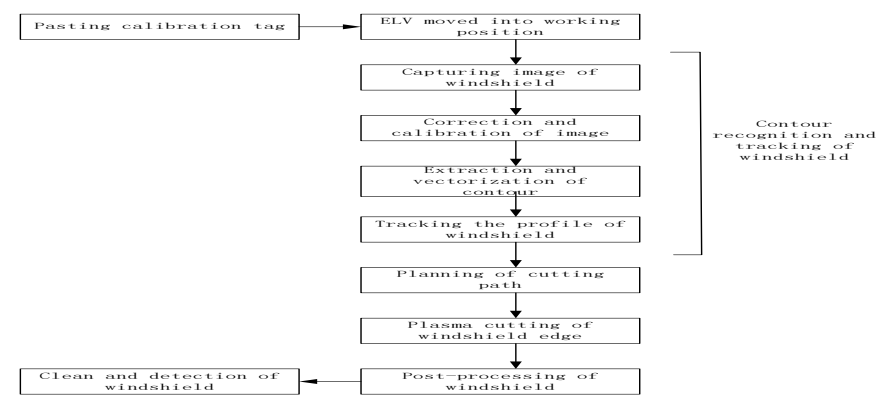

Fig. 1 Process system of generalized remanufacturing

Here, the concept of generalized remanufacturing (GR) is used for technology process analysis. GR refers to the whole process of repurchase, dismantling, repair, recycling and material utilization after the end of product utilization process, with the process system shown in Fig. 1. GR starts from the repurchase of waste products. Due to the geographical and temporal dispersion of waste products, the collection and repurchase of them is a very complex system, which depends on the support of the relevant technologies of reverse supply chain. After waste products are collected in processing enterprises, they are usually disassembled and cleaned. Since the conditions of waste products are different, and their brands and specification parameters are varied, large-scale processing is difficult. After disassembling, good-quality parts can be directly reused, for example, sold as repair parts. For parts with remanufacturing value, surface engineering technologies, such as cladding, plasma cladding, etc., can be used to repair them to restore their functions. After quality testing, they can be used as parts for new product manufacturing, or be sold directly in markets. Parts without remanufacturing value can be used as 
recycled materials in manufacturing process after crushing and sorting process, replacing a part of the virgin materials.

\section{HIERARCHY OF IR}

The relevant technologies and measures of IR play a more essential role in all aspects in GR, because the uncertainty of factors in GR is much greater than forward manufacturing process. IR is generally divided into several layers, including management decision-making layer, manufacturing execution layer, device control layer. They fuse relevant knowledge of artificial intelligence from the aspects of management decisionmaking, process control and device intelligent control. For remanufacturing process, a technology system of IR based on layer relationship is proposed in this study, which is shown in Fig. 2.
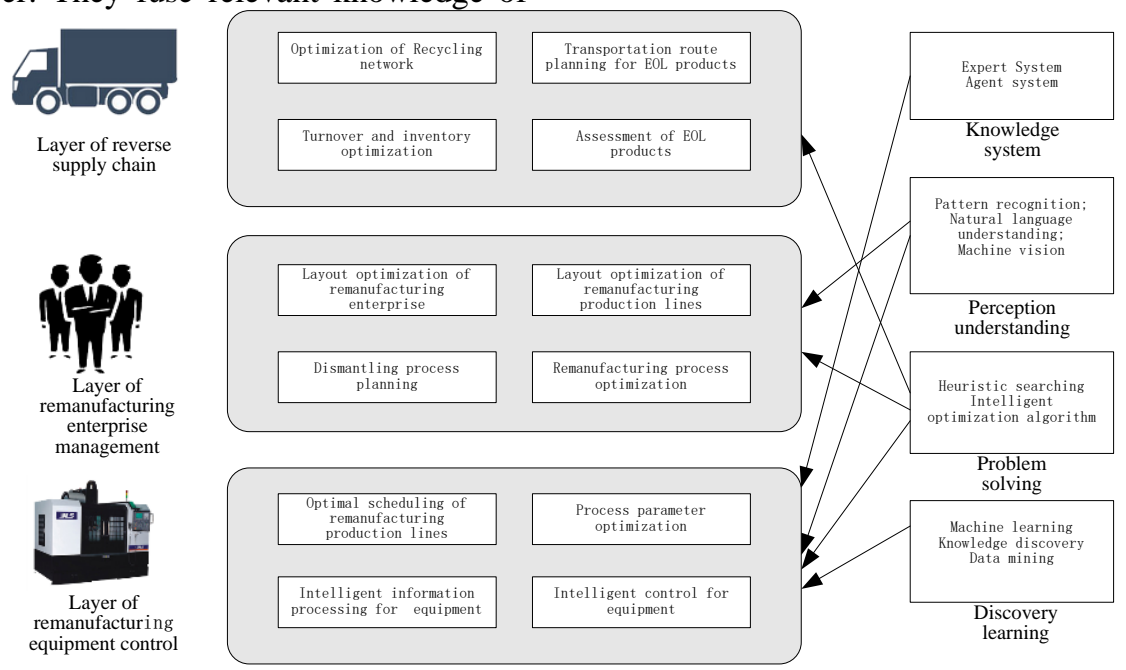

Fig. 2 Technology system of IR

IR is divided into three layers from macro to micro, namely, reverse logistics layer, remanufacturing enterprise management layer, remanufacturing equipment control layer. The top layer is reverse logistics layer. It not only includes the repurchase and transportation services of waste products, but also includes the transportation and turnover management of disassembled pieces in IR process, as well as the transportation and management of recycled materials. Moreover, the transportation and sale of remanufactured products also belong to this layer. The next layer is remanufacturing enterprise management layer. In this layer, optimization algorithms and technologies resorting to artificial intelligence can be used for the information processing and decision making of all aspects, from remanufacturing enterprise distribution points, the layout optimization of remanufacturing production lines, to the internal dismantling process planning and production process optimization of remanufacturing enterprises. The bottom layer is remanufacturing equipment control layer. In this layer, intelligent information process measures and intelligent control technologies are used to perform real-time detection and feedback control for the process parameters of remanufacturing equipment. The process parameters and the machining paths are calculated and planned in real time according to the diversity of remanufacturing products, and the online detection and intelligent information process is performed for the parts which need remanufacturing. The remanufacturing process is technically guaranteed to generate large-scale efficiency which is the same as normal forward manufacturing, enabling the remanufacturing enterprises create economic benefits at the same time of creating social benefits.

\section{A. Intelligence Technologies Oriented to Reverse Supply Chain of Remanufacturing}

For the reverse supply chain management oriented to remanufacturing, a large number of studies have been carried out by researchers at home and abroad. Among them, much attention has been paid to logistics network optimization or transportation path optimizing [2-4]. Intelligent optimization models and algorithms, including genetic algorithm, ant colony algorithm, Petri network model, etc., have been applied in this field. Moreover, branch and bound algorithm, heuristic algorithms have also been applied in supply chain layout and inventory management. From the current study, much studies on intelligent algorithms have been carried out based on supply chain network optimization and transportation route optimization, while there are few studies on reverse supply chains of waste products combining information tracking technology, things of Internet technology, E-commerce, etc. Even less is about reverse supply chains focusing on the features of specified waste products. Therefore, study on the reverse supply chain of waste products combining the hardware or software technologies of intelligent information processing and things of Internet is of great importance.

\section{B. Intelligent Technologies for Remanufacturing Business Management}

In this layer, the first problem to be solved is the planning and assessment of remanufacturing enterprises. The goal is to design optimal overall remanufacturing enterprise planning, such as the layout form of remanufacturing production lines, on 
the basis of sufficiently considering the market inventory of waste products and constraints, including investment, labor, land, etc. The key to this problem is to model different remanufacturing modes based on expert experience, and use intelligent optimization algorithms to solve them. Thus, the intelligent algorithm for multi-objective optimization has an important value in the field of remanufacturing.

The production scheduling and the optimization of production process planning of remanufacturing enterprises is also a possible field of intelligent technologies. This field mainly depends on human experience at present. However, with the expansion of production planning and production process data, the measures depending on manual operation dramatically affect production efficiency, which is typical in the dismantling process planning of scrapped automobiles. For small dismantling enterprises, production can depend on human experience. However, for large batch of ELV dismantling enterprises, under the condition that the beats of dismantling lines are extremely compact, production failures occur if just depending on human experience. This is not only because the technique levels of workers are uneven, but also because the classes of scrapped automobiles dismantled by large-scale dismantling enterprises are much more than those of small dismantling enterprises. Therefore, the production efficiency and management level of dismantling enterprises can be significantly improved by using computer information processing technologies and intelligent technologies to plan and manage the dismantling process of scrapped automobiles, and providing dismantling instructions to operatives.

\section{Intelligent Technologies Oriented to Remanufacturing Equipment}

Remanufacturing equipment includes dismantling equipment, detection equipment, surface repair equipment, part calibration equipment. Compared to normal forward manufacturing, remanufactured waste parts have relatively large differences in terms of geometric accuracy, surface quality, etc. [5], which requires that the remanufacturing equipment has higher flexibility and intelligence level. The intelligent technologies of remanufacturing equipment include the software or hardware technologies of intelligent information processing and intelligent control, etc. Taking the dismantling of windshields of scrapped automobiles as an example, since the types, sizes of scrapped automobiles are different, robot off-line programming technology which is the same as forward manufacturing is helpless. To deal with this, machine vision and special sensors, as well as contour track algorithm based on secondary track are used. The cutting path of windshield edge on body is calculated in real time for each scrapped automobile to be dismantled. Then, the whole windshield can be obtained by cutting the body using plasma heads.

The intelligent technologies of surface repair equipment are more essential parts. Special sensors are used to detect the regions to be repaired in real time. On the basis of processing and recognizing detection data, machine learning algorithms are combined to generate reasonable process parameters. Controllers are used to guarantee the repair accuracy of parts, guaranteeing the consistency of the quality of repaired parts [6].

\section{CASE STUDY}

Currently, in the ELV (end of life vehicle) dismantling industry, windshields are destructively removed at first, and then are sent to landfills as waste. The main reason is that the efficiency of manual dismantling is too low, causing the phenomenon that the value of glass cannot compensate the cost of dismantling. Moreover, the destructive removal makes the purity of the ensuing recycled materials low, which not only increases the difficulty of recycling, but also affects the reusing. If the windshields of ELV are non-destructively removed, pass security tests, and enter automobile maintenance enterprises through auto part logistics network, great economic value and social benefits can be created in the aspects of economical use of resources and energy saving.

Unlike the manufacturing process of automobiles, the brands and types of ELV processed on dismantling lines are random, it is impossible that there are only a few types as on automobile production lines. Therefore, under this condition, adopting offline programming or offline teaching mode to define cutting paths, and then controlling the automatic work of cutting equipment is unfeasible. Conversely, using artificial intelligence to improve the automation and flexibility is a suitable approach.

Thus, we propose a windshield edge cutting technology scheme based on artificial intelligence and machine vision. The detailed process is shown in Fig. 3. First, before the ELV enters the windshield cutting station, a calibration sticker is attached at the center of it. The sticker is printed according to predefined size and patterns. It is mainly used for the calibration of position and size parameters in vision measurement process. When the ELV pushed into the windshield cutting station, the camera on the top of equipment captures the profile image of windshields. The images are processed through image processing techniques and the edge image of windshields is finally obtained. Then, the basic parameters of the edge image can be derived according to the parameters of the sticker in the image. Finally, vectorization process is performed for the edge image to obtain the flat trajectory used in secondary tracking. Then, the edge of windshield is tracked and scanned by the tracking sensor installed at the end of multi-axis cutting equipment, obtaining the three dimensional geometric parameters of the windshield edge, and the cutting path of windshield on the body of ELV is created. Finally, with plasma cutting tool which mounted on the cutting equipment, the windshield of ELV can be disassembled for reusing. Fig.4 is the experiment for this study. 


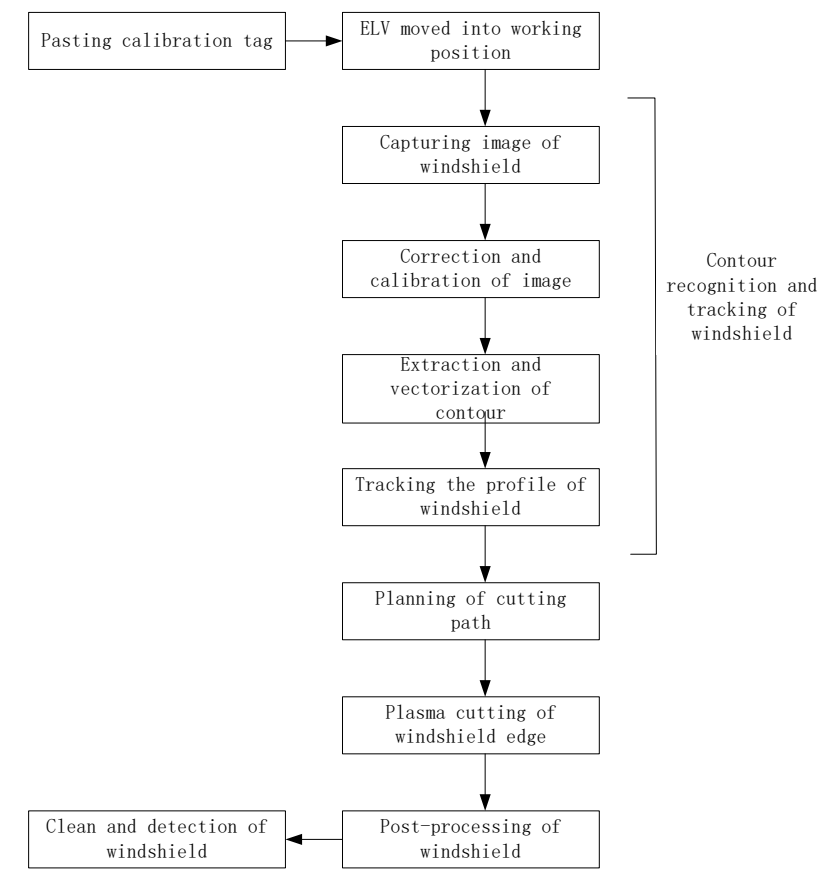

Fig. 3 Process of recycling windshield of ELV

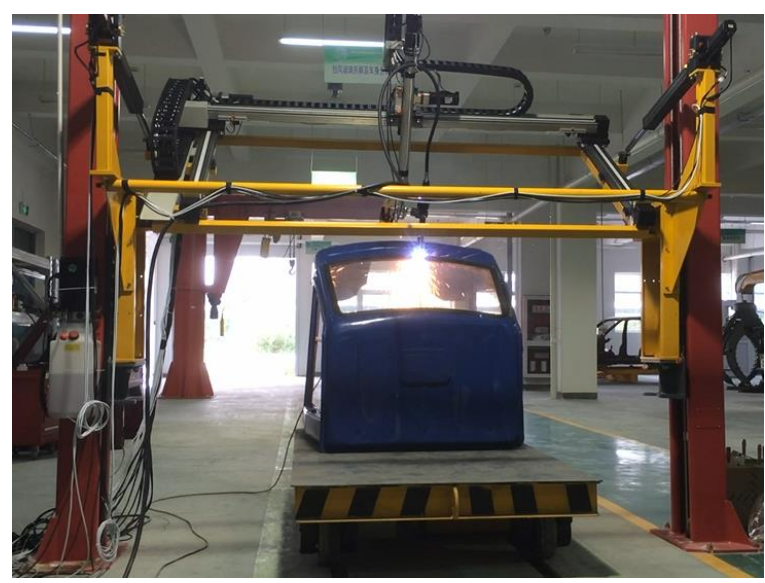

Fig. 4 Experiment of cutting windshield of ELV

\section{CONCLUSIONS}

It can be concluded from the above analysis that IR is to integrate relevant theories and technologies of artificial intelligent into remanufacturing field, in order to solve each kind of uncertain factors in remanufacturing field. It plays a vital role in the management and supply chain layer, disassembling, and remanufacturing processes. However, due to the limitation of artificial intelligent technologies themselves, in the detailed application process of these selflearning algorithms and intelligent optimization algorithms, the performance largely depends on the quality of the analysis and modeling of remanufacturing process. Moreover, many problems in process decision making are difficult to solve by only using artificial intelligent technologies. Therefore, from the current development, the relevant technology ideas of IR should be correctly treated. Not only should relevant technologies of IR be studied and developed, but also should the blind expansion be avoided, and the expectation that artificial intelligent measures can solve all problems in GR field should be given up.

\section{ACKNOWLEDGMENT}

This work was financially supported by Science \& Technology Pillar Program of Jiangsu Province (No. BE2013060). Key Project of Natural Science Research of Higher Education Institutions of Jiangsu Province (No. 15KJA460001). Postdoctoral Research Program of Jiangsu Province (No.1401071C).

\section{REFERENCES}

[1] Bingshi Xu, Shiyun Dong, Sheng Zhu, Peijing Shi. Prospects and Developing of Remanufacture Forming Technology [J]. JOURNAL OF MECHANICAL ENGINEERING, 2012.48(15):96-104.

[2] Bo Xing, Wen-Jing Gao, Kimberly Battle, etc. Artificial Intelligence in Reverse Supply Chain. http://arxiv.org/ftp/arxiv/papers/1012/1012.4046.pdf.

[3] Muthusamy Aravendan1, Ramasamy Panneerselvam.Literature Review on Network Design Problems in Closed Loop and Reverse Supply Chains[J]. Intelligent Information Management, 2014, 6, 104-117.

[4] Qiong Liu, Jingjing Ye, Xingyu Shao. Design of logistics networks for manufacture / remanufacture in uncertain environment $[\mathrm{J}]$. Journal of Huazhong University of Science and Technology. (Nature Science Edition), 2007.35(10):80-83.

[5] Jing Zhang, Gongqian Liang. Reliability Analysis of Remanufacturing Production System under Part Quality Uncertainty [J]. Industrial Engineering Journal, 2013.16(2):117-121.

[6] Rui Liu, Qingfeng Du. Recognition of Surface Defects in Laser Remanufacturing Robot [J]. LASER JOURNAL, 2015.36(9):60-63. 